\title{
Standardization of PSOAS Muscle Index Measurements Using Computed Tomography
}

\author{
P. Lakshmi Prashanthi ${ }^{1}$, Rajoo Ramachandran ${ }^{2}$, Adhilakshmi ${ }^{3}$, Prabhu Radhan ${ }^{4}$, Venkata Sai ${ }^{5}$ \\ ${ }^{1} J u n i o r$ Resident, Department of Radiology, ${ }^{2}$ Associate Professor, Department of Radiology, ${ }^{3}$ Allied Health Science Junior \\ Resident, Department of Radiology, ${ }^{4}$ Associate Professor, Department of Radiology, ${ }^{5} \mathrm{Head}$ of Department, Department of \\ Radiology, Sri Ramachandra Institute of Higher Education and Reasearch, India
}

Corresponding author: P. Lakshmi Prashanthi, No 1 Ramachandra nagar, SRIHER, Porur, Chennai-600116, iNDIA

DOI: http://dx.doi.org/10.21276/ijcmsr.2020.5.1.38

(c) BY-NC-ND

How to cite this article: P. Lakshmi Prashanthi, Rajoo Ramachandran, Adhilakshmi, Prabhu Radhan, Venkata Sai. Standardization of PSOAS muscle index measurements using computed tomography. International Journal of Contemporary Medicine Surgery and Radiology. 2020;5(1):A169-A172.

\section{A B S T R A C T}

Introduction: Sarcopenia is defined as loss of muscle mass less than two standard deviations from the normal reference value. It has gained importance in the prognostication of patients with chronic illness such as cirrhosis and cancer. Quantification of skeletal muscle has been done by various methods but cross sectional imaging remains gold standard. Study aimed to calculate Psoas muscle index in normal patients.

Material and Methods: In our study 100 patients who underwent CT with no obvious co-morbidities for donor status evaluation were selected and total Psoas muscle area (TPA) is measured which is then standardized for height to get the Psoas muscle index (PMI).

Results: It was observed that mean psoas muscle index decreased significantly with increasing age.

It was also observed that mean psoas muscle index in female population was comparatively less than mean psoas muscle index in male population.

Conclusions: Standardization of mean psoas muscle index enables evaluating normal and abnormal mean psoas muscle index thereby, predicting potential sarcopenia and initiate appropriate patient management / intervention. This can be used for predicting morbidity and mortality in cirrhosis, lung cancer, colorectal cancer patients.

Keywords: Sarcopenia, Psoas Muscle Index, Total Psoas Muscle Area, Skeletal Muscle Index, Cirrhosis, Cut Off Value.

\section{INTRODUCTION}

The loss of skeletal muscle mass is sarcopenia. ${ }^{1}$ Measuring sarcopenia is used to estimate the prognosis in patients with cirrhosis, gall bladder cancer, lung cancer and colorectal cancer. ${ }^{2-6}$ Sarcopenia is one of the most common complication in these patients. Measuring sarcopenia as an index related to survival of these patients lack optimal index in terms of objectivity, practicality, reproducibility and prognostic performance. Cross-sectional abdominal imaging is a promising tool for quantifying skeletal muscle mass and by assessing sarcopenia it also helps to identify adipose tissue infiltration. Skeletal muscle quantification is done in various countries, however because of the variability in body size, life styles and ethnicity ${ }^{7-10}$ there has been no standardized value for Indian population. This study aimed to standardize the psoas muscle mass measurement which is considered as an index measurement for assessing skeletal muscle mass. Our study consists of 100 patients who had underwent CT with no obvious comorbidities for donor status evaluation were selected and total Psoas muscle area (TPA) was measured which was then standardized for height to get the Psoas muscle index (PMI).

\section{MATERIAL AND METHODS}

100 patients were referred to CT examination from various departments from Sri Ramachandra Medical Centre, Porur, Chennai-600 116. Philips brilliance CT 16-slice multi detector high performance system suited for all examination of the abdomen studies.

\section{Methodology}

After obtaining ethical clearance from our institution our study was performed as a retrospective. We included a sample size of 100 normal patients in the study period between June 2018 to march 2019 at department of radiology and imaging sciences, Sri Ramachandra Medical Centre, Porur, Chennai-600 116.

Inclusion criteria: Patients referred for CT abdomen in the age group of 21-70 years for donor evaluation status.

Exclusion criteria: Patients with scoliosis, Chronic debilitating illness like diabetes, infections, smokers, acute abdomen with excessive gaseous distension and pregnant patients.

CT protocol included a unique hospital ID of the patient and the procedure was well explained. Patient was positioned 
in supine position, feet first orientation with Xiphisternum as the landmark. Abdomen routine protocol was selected from the protocol library. First a single AP scout film was taken. The scan type is a helical scan type with $2 \mathrm{~mm}$ acquisition at $0.75 \mathrm{~mm}$ reconstruction with pitch factor $0.938: 1$, rotation time $0.5 \mathrm{sec}, 120 \mathrm{kV} / 200 \mathrm{mAs}$, detector coverage $24 \mathrm{~mm}$, coverage speed $45 \mathrm{~mm} / \mathrm{sec}$, total exposure time $5-8 \mathrm{sec}$ and a large SFOV.

\section{Procedure}

After positioning the patient on the CT table and appropriate immobilization measures were taken to prevent patient fall and also to avoid motion artefacts. CT with the above mentioned protocols were carried out with anatomical coverage extending from diaphragm to symphysis pubis. The scan was obtained during a single breath hold for 5-9secs approximately. Image reconstruction from the acquired image data were performed with an parameters: effective slice thickness of $0.75 \mathrm{~mm}$ (retrospective reconstruction). The data sets were transferred to a Philips advanced workstation and post-processing of the images namely multiplanar reconstruction (MPR) were performed

\section{Measurement protocol}

CT images were transferred to an Advantage Windows workstation (ADW 4.4, GE Healthcare) and psoas muscle areas were assessed using Advantage Windows Area Analysis software.

\section{Technique}

Step 1: Select the axial image at the level of L3 vertebrae for Area analysis at which both the transverse process of the vertebrae at equidistant to each other
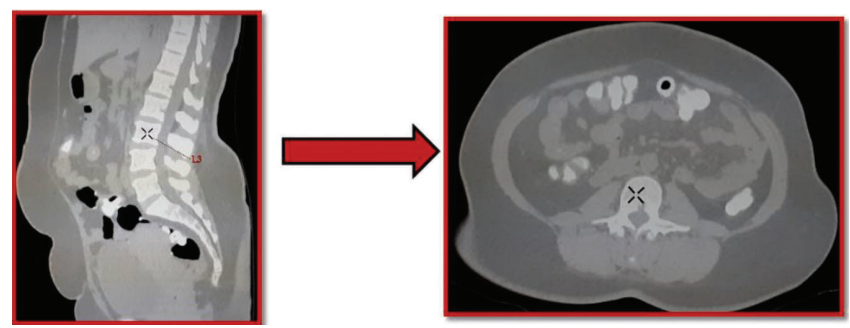

Step 2: Click the segment menu and select the threshold icon. Adjust the threshold from -30 to +110 HU. This enabled automatic calculation of psoas muscle area by excluding vasculature and areas of fatty infiltration based on Hounsfield unit (HU)

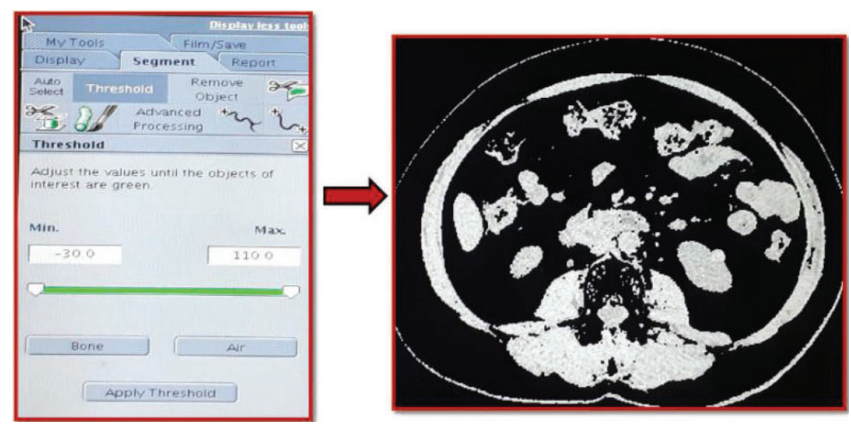

Step 3: Select the apply icon and select the free form ROI icon.Segmentation of the psoas muscle was performed by using a line tracing of a free form ROI on the selected slice and then select apply icon

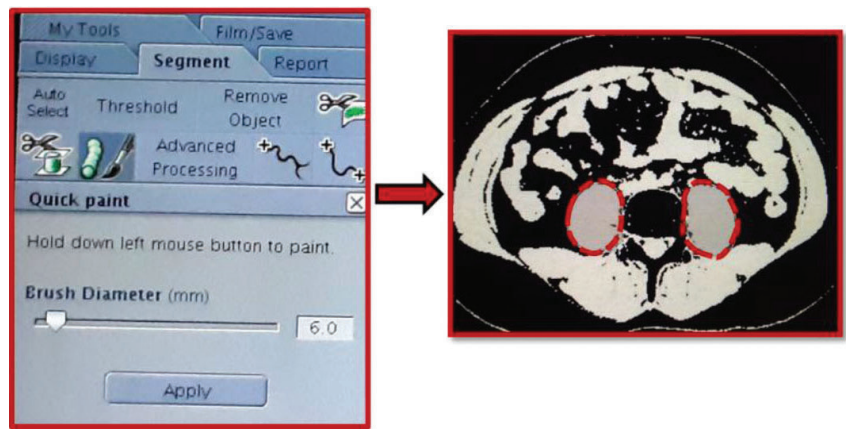

Step 4: Click on to display menu and select the area icon. Click the right psoas muscle on the window and the outline is drawn to measure the area.Subsequently measure the left Psoas muscle and calculate the total psoas area

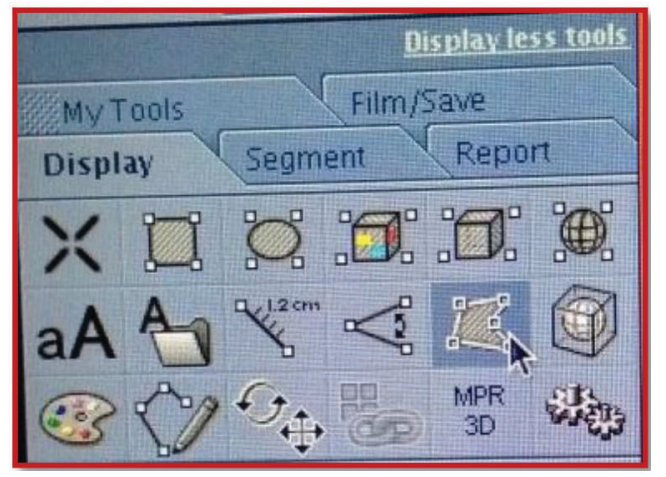

The total psoas area was then normalized for height as per convention for body composite measurements (TPA in square millimeter / square meter $)^{8-9}$

\section{RESULTS}

This retrospective study of standardizing psoas muscle area in Indian population included 100 normal people. Of these 100 normal patients, 66 were males and 34 were females (table-1).

Our study included 8 female and 12 male patients under 20-30 year age group, 7 female and 13 male under $31-40$ year age group, 6 female and 14 male patients under $41-50$ age group, 9 female and 11 male patients under $51-60$ age group, 4 female and 16 male patients under $61-70$ years age group.

The table-2 shows the mean Psoas muscle index of females with respect to age group in our study group. It can be seen that the mean Psoas muscle index decreases as the age increases.

The table- 3 shows the mean Psoas muscle index of males with respect to age group in our study group. It can be seen that the mean Psoas muscle index decreases as the age increases The graph-1 shows the mean Psoas muscle index of males being higher than females.

\begin{tabular}{|l|c|}
\hline Sex & Number of patients \\
\hline Male & 66 \\
\hline Female & 34 \\
\hline Total & 100 \\
\hline \multicolumn{2}{|c|}{ Table-1: Gender Distribution } \\
\hline
\end{tabular}




\begin{tabular}{|l|c|c|c|c|c|c|}
\hline Age group & No of patients & Sex & $\begin{array}{c}\text { Height } \\
(\mathbf{M})\end{array}$ & $\begin{array}{c}\text { RT Psoas area } \\
\left(\mathbf{m m}^{2}\right)\end{array}$ & $\begin{array}{c}\text { LT Psoas Area } \\
\left(\mathbf{M m}^{\mathbf{2}}\right)\end{array}$ & $\begin{array}{c}\text { PMI } \\
\left(\mathbf{m m}^{\mathbf{2}} / \mathbf{m}^{\mathbf{2}}\right)\end{array}$ \\
\hline $21-30$ & 8 & $\mathrm{~F}$ & 1.53 & 807.38 & 802.76 & 782.76 \\
\hline $31-40$ & 7 & $\mathrm{~F}$ & 1.52 & 666.14 & 734.11 & 605.86 \\
\hline $41-50$ & 6 & $\mathrm{~F}$ & 1.57 & 636.9 & 664.06 & 522.65 \\
\hline $51-60$ & 9 & $\mathrm{~F}$ & 1.59 & 538 & 548.43 & 415.79 \\
\hline $61-70$ & 4 & $\mathrm{~F}$ & 1.46 & 512.05 & 559.8 & 408.10 \\
\hline \multicolumn{2}{|r|}{ Table-2: Psoas muscle index of Female patients with respect to age group } \\
\hline
\end{tabular}

\begin{tabular}{|c|c|c|c|c|c|c|}
\hline Age group & No of patients & Sex & $\begin{array}{l}\text { Height } \\
\text { (m) }\end{array}$ & $\begin{array}{c}\text { RT Psoas area } \\
\left(\mathrm{mm}^{2}\right)\end{array}$ & $\begin{array}{c}\text { LT psoas } \\
\text { area }\left(\mathrm{mm}^{2}\right)\end{array}$ & $\begin{array}{c}\text { PMI } \\
\left(\mathrm{mm}^{2} / \mathrm{m}^{2}\right)\end{array}$ \\
\hline $21-30$ & 12 & $M$ & 1.67 & 1076.62 & 1114.15 & 840.30 \\
\hline $31-40$ & 13 & $M$ & 1.61 & 1044.0 & 1068.0 & 825.53 \\
\hline $41-50$ & 14 & $\mathrm{M}$ & 1.66 & 1013.82 & 1024.81 & 722.68 \\
\hline $51-60$ & 11 & $\mathrm{M}$ & 1.48 & 878.0 & 826.1 & 642.57 \\
\hline $61-70$ & 16 & $M$ & 1.65 & 674.7 & 700.67 & 492.28 \\
\hline
\end{tabular}

\begin{tabular}{|l|l|c|c|}
\hline Age group & Sex & Mean & Standard deviation \\
\hline \multirow{2}{*}{$21-30$} & $\mathrm{~F}$ & 805.07 & 479.83 \\
\cline { 2 - 4 } & $\mathrm{M}$ & 840.3 & 214.03 \\
\hline \multirow{3}{*}{$31-40$} & $\mathrm{~F}$ & 605.86 & 185.27 \\
\cline { 2 - 4 } & $\mathrm{M}$ & 825.53 & 245.00 \\
\hline \multirow{3}{*}{$41-50$} & $\mathrm{~F}$ & 560.85 & 196.67 \\
\cline { 2 - 4 } & $\mathrm{M}$ & 722.68 & 217.99 \\
\hline \multirow{2}{*}{$51-60$} & $\mathrm{~F}$ & 415.79 & 83.89 \\
\cline { 2 - 4 } & $\mathrm{M}$ & 642.57 & 142.04 \\
\hline $61-70$ & $\mathrm{~F}$ & 408.01 & 100.83 \\
\cline { 2 - 4 } & $\mathrm{M}$ & 492.28 & 129.77 \\
\hline
\end{tabular}

Table-4: Represents the mean and standard deviation with respect to age and gender

\begin{tabular}{|l|c|c|}
\hline Age group & $\begin{array}{c}\text { Normal reference } \\
\text { value for females } \\
\left(\mathbf{m m}^{2}\right)\end{array}$ & $\begin{array}{c}\text { Normal reference } \\
\text { value for males } \\
\left(\mathbf{m m}^{2}\right)\end{array}$ \\
\hline $21-30$ & $472-1139$ & $719-961$ \\
\hline $31-40$ & $468-743$ & $692-986$ \\
\hline $41-50$ & $403-719$ & $608-837$ \\
\hline $51-60$ & $397-437$ & $558-727$ \\
\hline $61-70$ & $309-507$ & $429-559$ \\
\hline \multicolumn{2}{|c|}{ Table-5: Represents 95\% confidence interval in males and } \\
females \\
\hline
\end{tabular}

The table- 4 shows mean and standard deviation of Psoas muscle index with respect to gender and age.

The table- 5 shows by calculating standard deviation for each age group among males and females a $95 \%$ confidence interval was obtained which represents that any patient having Psoas muscle index less than the lower limit is considered to be sarcopenic.

\section{DISCUSSION}

The term Sarcopenia had been proposed by Rosenberg ${ }^{1}$ in 1989 as age related muscle atrophy that appears in combination with low muscle strength and/or physiological performance condition. The European Working Group On

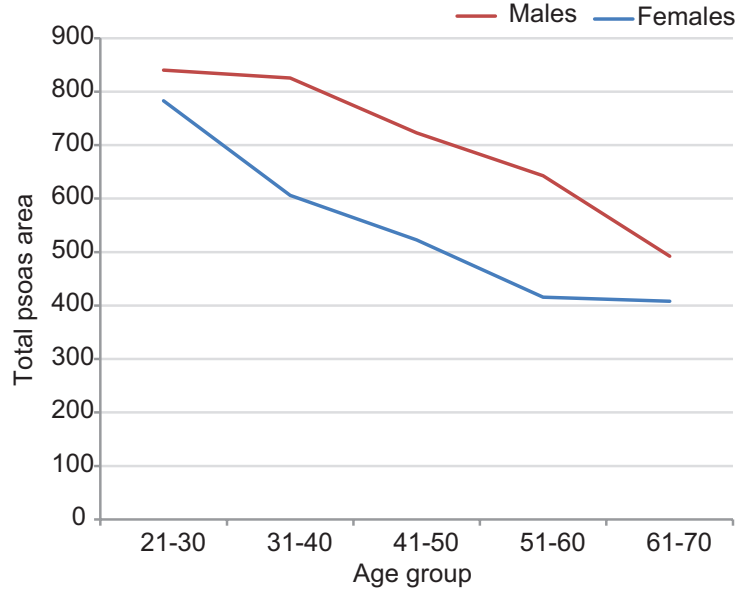

Figure-1: Represents the comparison between the Psoas muscle index and the age group Between males and females

Sarcopenia in Older People defined sarcopenia (EWGSOP) as a syndrome and further described $\mathrm{CT}$ and MRI as the modalities of choice as it delineates fat infiltration. ${ }^{1}$

This retrospective study was conducted to calculate the skeletal muscle mass by measuring the age specific and sex specific mean psoas muscle index of Indian population and thereby standardizing it, so that it can be used as a screening tool in prediction and management of sarcopenic patients.

Our study included 100 normal patients, out of which 66 were males and 34 were females. (table-1). The age distribution ranged from 20 to 70 years. Our study included 8 female and 12 male patients under 20-30 years age group, 7 female and 13 male under 31 - 40 years age group, 6 female and 14 male patients under $41-50$ years age group, 9 female and 11 male patients under $51-60$ years age group, 4 female and 16 male patients under $61-70$ years age group.

The psoas muscle was taken as a representative muscle to measure the skeletal muscle mass as it is seen to be unaffected by activity. ${ }^{10-11}$

The mean psoas muscle index values can be used to compare and assess the normal and abnormal psoas muscle area. The present study revealed that mean psoas muscle index varied 
with varying age group. ${ }^{12,13}$ The mean psoas muscle index decreased as the age increased mirroring the fact that the skeletal muscle mass decreases with aging. ${ }^{14-16}$ It was observed that mean psoas muscle index decreased significantly with increasing age(Ref: table 2, table 3).

As per Asian Working Group for Sarcopenia (AWGS) recommendations and other previous studies sarcopenia is defined as two standard deviations less than the normal value $^{12-14}$ which has been applied in this study (table4,table5). Moreover, it was observed that mean psoas muscle index in female population was comparatively less than mean psoas muscle index in male population (figure-1). This standardized mean psoas muscle index can be employed to predict sarcopenia in symptomatic patients by comparing the mean psoas muscle index of the individual with standardized values and initiate prompt management since sarcopenia can be one of the most important cause of functional decline and loss of independence in adults.

Limitation of our study was other indices for body mass measurement could not be done as it was a retrospective study.

\section{CONCLUSION}

Standardization of mean psoas muscle index enables evaluating normal and abnormal mean psoas muscle index thereby, predicting potential sarcopenia and initiate appropriate patient management / intervention. This can be used for predicting morbidity and mortality in cirrhosis, lung cancer, colorectal cancer patients.

\section{REFERENCES}

1. Cruz-Jentoft AJ, Baeyens JP, Bauer JM, et al. Sarcopenia: European consensus on definition and diagnosis: Report of the European Working Group on Sarcopenia in Older People. Age Ageing. 2010;39(4):412-423.

2. Prado CM, Lieffers JR, McCargar LJ, et al. Prevalence and clinical implications of 287 sarcopenic obesity in patients with solid tumours of the respiratory and 288 gastrointestinal tracts: a population-based study. Lancet Oncol 2008; 9(1): 629-635.

3. van Vledder MG, Levolger S, Ayez N, Verhoef C, Tran TC, Ijzermans JN. Body 290 composition and outcome in patients undergoing resection of colorectal liver 291 metastases. Br J Surg 2012; 99(5): 550-557. 292

4. Peng P, Hyder O, Firoozmand A, et al. Impact of sarcopenia on outcomes following 293 resection of pancreatic adenocarcinoma. J Gastrointest Surg 2012; 16(6): 1478-1486. 294

5. Kaido T, Ogawa K, Fujimoto Y, et al. Impact of sarcopenia on survival in patients undergoing living donor liver transplantation. Am J Transplant 2013; 13(3): 1549- 2961556.

6. Meza-Junco J, Montano-Loza AJ, Baracos VE, et al. Sarcopenia as a prognostic 298 index of nutritional status in concurrent cirrhosis and hepatocellular carcinoma. J 299 Clin Gastroenterol 2013; 47(1): 861-870.

7. Chen LK, Liu LK, Woo J, et al. Sarcopenia in Asia: consensus report of the Asian 304 Working Group for Sarcopenia. J Am Med Dir Assoc 2014; 15(5): 95-101.

8. Mourtzakis M, Prado CM, Lieffers JR, Reiman T,
McCargar LJ, Baracos VE. A practical and precise approach to quantification of body composition in cancer patients using computed tomography images acquired during routine care. Appl Physiol Nutr Metab. 2008; 33(5):997-1006

9. Dodson RM, Firoozmand A, Hyder O, et al. Impact of sarcopenia on outcomes following intra-arterial therapy of hepatic malignancies. J Gastrointest Surg. 2013;17(12):2123-2132.

10. Fiber types in mammalian skeletal muscles, Schiaffino S, Reggiani C, Physiol Rev. 2011; 91(4):1447-53.

11. Dasarathy S, Merli M. Sarcopenia from mechanism to diagnosis and treatment in liver disease. J Hepatol. 2016;65(6):1232-1244.

12. Kim J, S, Kim W, Y, Park H, K, Kim M, C, Jung W, Ko B, S: Simple Age Specific Cutoff Value for Sarcopenia Evaluated by Computed Tomography. Ann Nutr Metab 2017;71(2):157-163.

13. Hamaguchi Y, Kaido T, Okumura S, Kobayashi A, Hammad A, Tamai Y, Inagaki N, Uemoto S, Proposal for new diagnostic criteria for low skeletal muscle mass based on computed tomography imaging in Asian adults, Nutrition (2016), doi: 10.1016/j.nut.2016.04.003.

14. Chen LK, Liu LK, Woo J, et al. Sarcopenia in Asia: consensus report of the Asian 304 Working Group for Sarcopenia.J Am Med Dir Assoc 2014; 15(3): 95-101.

15. Lexell J, Taylor CC, Sjöström M. What is the cause of the ageing atrophy? Total 315 number, size and proportion of different fiber types studied in whole vastus lateralis 316 muscle from 15- to 83-year-old men. J Neurol Sci 1988; 84(6): 275-294. 317

16. Frontera WR, Hughes VA, Fielding RA, Fiatarone MA, Evans WJ, Roubenoff R. 318 Aging of skeletal muscle: a 12-yr longitudinal study. J Appl Physiol (1985) 2000; 319 88: 1321-1326.

Source of Support: Nil; Conflict of Interest: None

Submitted: 14-01-2020; Accepted: 01-02-2020; Published online: 29-02-2020 